\title{
Help-seeking behaviour and its impact on patients attending a psychiatry clinic at National Hospital of Sri Lanka
}

\author{
DM Gomez, C Gunarathna, S Gunarathna, K Gnanapragasam, R Hanwella
}

\begin{abstract}
Background Mental illness contributes significantly to the global disease burden. There is great diversity in the manner in which mentally ill patients seek help as this is influenced by their beliefs and the opinion of the familysocial support unit. The stigma associated with mental illness is a barrier to effective therapy in Sri Lanka where systematic public awareness programmes are minimal.
\end{abstract}

Objective To study the help-seeking behaviour and its impact on patients attending a psychiatry clinic of the National Hospital of Sri Lanka.

Methods A cross sectional study was carried out among 120 attendees of the psychiatry clinic of the National Hospital of Sri Lanka. Sample was selected using systematic sampling. Data was collected using an interviewer administered questionnaire.

Results More than half the participants sought psychiatric care as their first help-seeking behaviour and found it significantly more useful than non-psychiatric care alternatives. The average time to seek psychiatric care, irrespective of the pathway to care, was less than one month. The recommendation of the family and the social support unit and perceiving that the symptoms were due to a mental illness were the key factors in determining helpseeking behaviour. The average expense on alternative care was zero. There was no significant difference on the impact to employment among those that chose psychiatry care initially from those that did not.

Conclusions Our findings suggest that mentally ill patients presenting to a tertiary care hospital in Colombo, are likely to seek psychiatric care early. This is probably due to better recognition and knowledge regarding available treatment.

Ceylon Medical Journal 2017; 62: 222-27

DOI: http://doi.org/10.4038/cmj.v62i4.8571

\section{Introduction}

Mental illness comprises a broad range of illnesses, with different symptoms. They are generally characterized by abnormalities in thoughts, emotions, behaviour and relationships with others. Most mental illness can be successfully treated [1].

Mental illnesses contribute significantly to the overall global disease burden, affecting more than 450 million people worldwide. According to a recent WHO estimate, nearly one third of all years lived with disability (YLDs) can be attributed to neuropsychiatric conditions [2]. In Sri Lanka, neuropsychiatric disorders are estimated to contribute to $11.5 \%$ of the global burden of disease [3]. About $5-10 \%$ of the Sri Lankan population is thought to suffer from mental illnesses requiring medical attention [4].

The Mental Health Policy of Sri Lanka was approved in 2005 and there is a mental health component in the General Health Policy of Sri Lanka. There is only one psychiatrist for 500,000 people in the country [4]. Although a referral system to secondary and tertiary health care settings exists execution of mental health directives to the public falls short [3]. Insufficient funding, lack of trained personnel and overreliance on the tertiary health care system are administrative barriers for providing accessible mental health services.

Help-seeking behaviour of patients are diverse and depend on several personal and disease related factors. Patients may choose to either ignore their symptoms, seek family or religious support, engage in traditional healing or alternate medicine practices, consult a Western medical practitioner who is not a psychiatrist or directly consult a psychiatrist. There is usually a considerable delay between the initial manifestation of symptomsand seeking help from a psychiatrist [4]. 
The associated stigma and lack of awareness about available treatment options for mental illness prevent many from accessing medical help. The general public has not been adequately educated that mental illnesses can be treated [4].

One study has investigated the traditional treatment methods followed by mentally ill patients in Sri Lanka. [5]. This however did not look at the impact of such behaviours on the patient. The present study aims to study the help-seeking behaviour and its impact on patients attending a psychiatry clinic in the tertiary care National Hospital of Sri Lanka in Colombo. This knowledge would prove invaluable to improve the health care services for patients with mental illness and plan systematic awareness programs for the community.

\section{Methods}

This cross-sectional study was carried out among 120 patients attending the University Psychiatry Unit, out-patient psychiatry clinic of the National Hospital of Sri Lanka. Patients were selected using systematic sampling.

All patients included in the study were followed up in the clinic and were over 12 years of age. Patients were excluded from the study if they were confused or were unable to understand or respond to questions or were below 18 years of age and a parent or legal guardian was not available.

A pilot study was conducted by the principal investigators prior to definitive data collection. The study instrument used was a purpose designed semi-structured interviewer administered questionnaire that gathered socio-demographic data, illness data, information on relevant help-seeking behaviour, beliefs and attitudes that determined these behaviours and the impact due to alternate care pathways regarding the first episode of illness in terms of financial cost and impact on the occupational capacity of the patient.

Ethical clearance was obtained from the Ethics Review Committee of the Faculty of Medicine, Colombo (CSRP/15/Gr.2/014 (AL 2011). Written informed consent was obtained from all patients. Relevant administrative clearance was obtained.

Data entry and analysis were performed using SPSS 20 software.

\section{Results}

\section{Socio-demographic characteristics}

The socio-demographic characteristics of the sample is shown in Table 1. A total of 120 individuals were included in the sample. There were 49 males $(40.8 \%)$. Majority were Sinhalese and Buddhist and were resident in the Colombo District.

\section{Help-seeking behaviour of patients}

The help-seeking behaviour of patients and the pathway taken to reach the psychiatry clinic is shown in Figure 1. Initial help was sought from traditional healers $(n=23)$, alternative medical practitioners (Ayurveda) $(\mathrm{n}=3)$, Western medical practitioners who was not psychiatrists $(n=29)$ and consultant psychiatrists $(n=65)$. All 29 who sought help from a Western medical practitioner (non psychiatrist) were subsequently referred to a specialist psychiatrist. Thirteen of the 23 who initially sought help from traditional healers also subsequently sought help from a specialist psychiatrist as the second point of contact. Others sought help from various sources before contacting specialized psychiatric services.

Sixty nine $(57.2 \%)$ participants found their first choice of help to be useful regardless of the type of treatment. Fifty six out of the $69(86.2 \%)$ had consulted a psychiatrist. Of those who sought non-psychiatric help only $13(23.1 \%)$ found it to be useful. Twenty one $(91.3 \%)$ of those who sought traditional or faith healing were dissatisfied with the treatment received and claimed it did not relieve symptoms.

\section{Time taken to access specialised psychiatric care}

Sixty two $(51.7 \%)$ sought help within a month after the initial onset of symptoms of mental illness while 29 (24.2\%) took more than 6 months. The remaining 29 sought help within one to six months.

Within the first month of the onset of symptom, 34 had consulted a psychiatrist directly and a further 13 had seen a psychiatrist after seeking alternative therapies. Most had taken less than one month to consult a psychiatrist.

\section{Beliefs and attitudes about the illness}

The perceived cause of symptoms experienced in the first episode of mental illness is shown in Table 2. Majority $(n=73 ; 60.8 \%)$ perceived that their symptoms were caused by a mental illness while $20(16.7 \%)$ believed it was due to a physical illness.

Of the 73 that thought that their symptoms were due to a mental illness $67.1 \%$ consulted a psychiatrist while $17.8 \%$ consulted a Western medicine practitioner (non psychiatrist). Of those who consulted a psychiatrist $44.6 \%$ did so because of recommendation by a family member or friend $(44.6 \%)$.

Traditional and faith healing was sought by $61.1 \%$ of those who believed the symptoms were due to supernatural causes. Interestingly $13.7 \%$ who thought they had a mental illness also sought help from traditional and faith healers.

The reasons for delay in consulting a psychiatrist and what prompted their consultation at a later point is shown in Table 3. In 82 the time taken to consult a psychiatrist was more than one month. Among them 27 (32.2\%) did not seek help from a psychiatrist because they did not believe they had a psychiatric illness. Seventeen (20.8\%) thought that the symptoms would resolve naturally. 
Table 1. Prevalence of fluorosis among sample of school children

\begin{tabular}{ll}
\hline & Number (\%) \\
\hline Gender & $49(40.8)$ \\
Males & $7(5.8)$ \\
\hline Age distribution & $43(35.8)$ \\
11-20 years & $49(40.8)$ \\
21-40 years & $21(17.6)$ \\
$41-60$ years & \\
$>60$ years & $100(83.3)$ \\
\hline Ethnicity & $9(7.5)$ \\
Sinhalese & $11(9.2)$ \\
Tamil & \\
Muslim & $81(67.5)$ \\
\hline Religion & $22(18.3)$ \\
Buddhism & $6(5.0)$ \\
Christianity & $11(9.2)$ \\
Hinduism &
\end{tabular}

\begin{tabular}{ll}
\hline Residence & $79(65.8)$ \\
Colombo & $25(20.8)$ \\
Gampaha & $8(6.7)$ \\
Kalutara & $8(6.7)$ \\
Others & \\
\hline
\end{tabular}

\begin{tabular}{ll}
\hline Educational qualifications & \\
No schooling & $1(0.8)$ \\
Grade 1-5 & $10(8.3)$ \\
Grade 6-11 & $44(36.7)$ \\
GCE O Level & $33(27.5)$ \\
GCE A Level & $6(5.0)$ \\
Tertiary education & $8(6.7)$ \\
\hline Marital Status & \\
Married & $69(57.5)$ \\
Unmarried & $47(39.2)$ \\
Divorced & $4(3.3)$ \\
\hline Living arrangements & \\
Lives alone & $2(1.7)$ \\
With parents and siblings & $40(33.3)$ \\
With spouse and children & $77(64.2)$ \\
Lives in an institution & $1(0.8)$ \\
\hline
\end{tabular}

\section{Impact of the delay in seeking treatment}

The majority $(\mathrm{n}=29 ; 52.7 \%)$ of those who sought alternative pathways to care incurred no expense. The rest had spent amounts ranging from Rs. 300 to Rs. 200,000, while six participants (10.9\%) had spent more than Rs. 50,000 .
Table 2. Perceived cause of symptoms experienced in the first episode

\begin{tabular}{ll}
\hline Cause & Number (\%) \\
\hline Mental illness & $73(60.8)$ \\
Physical illness & $20(16.7)$ \\
Supernatural causes & $18(15.0)$ \\
Environmental causes & $4(3.3)$ \\
and life stressors & \\
Not known & $5(4.2)$ \\
Total & 120 \\
\hline
\end{tabular}

Financial aid from family members and relatives was the main source of money for medical expenses $(63.6 \%)$. Personal income was used by $16(29.1 \%)$ participants. None of the participants had taken bank loans, sold assets or borrowed money to pay for alternative therapies. Two participants admitted to mortgaging household items.

Majority ( $\mathrm{n}=63 ; 52.5 \%)$ of the participants were employed at the time of onset of symptoms. Thirty one (83.8\%) who had sought psychiatry care initially and 23 $(88.5 \%)$ who sought alternative treatment were not able to continue their previous employment.

\section{Discussion}

Our objective was to study the help-seeking behaviour and its impact on patients attending a psychiatry clinic at the National Hospital of Sri Lanka. We found that more than half the patients had consulted a psychiatrist as their first help-seeking behaviour and close to a quarter of the respondents had seen a Western medical practitioner (non psychiatrist).

Although a large percentage had sought help from specialized psychiatry services and Western medical practitioners this finding cannot be generalized because the sample consisted of patients attending an out-patient psychiatry clinic in a tertiary hospital in Colombo. This group is more likely to seek treatment from a psychiatrist initially.

The findings of this study contrasts with a study conducted in the same clinic in 2004. That study reported that $51.9 \%$ of patients sought traditional help during the course of their illness, 9.1\% sought help from Ayurvedic practitioners and $25.9 \%$ sought magico-religious help [5]. However this study reported help-seeking during the entire course of the illness not just during the first episode of illness. However, it is possible that during the last decade, the pattern of help-seeking behaviour in patients with mentally illness in Sri Lanka has changed from one that was dominated by traditional healing methods to one that of seeking help from psychiatric services.

Similar findings have been reported from North India where $45.5 \%$ of first contact was a psychiatrist, $44 \%$ sought help from a non-psychiatric physician while only 


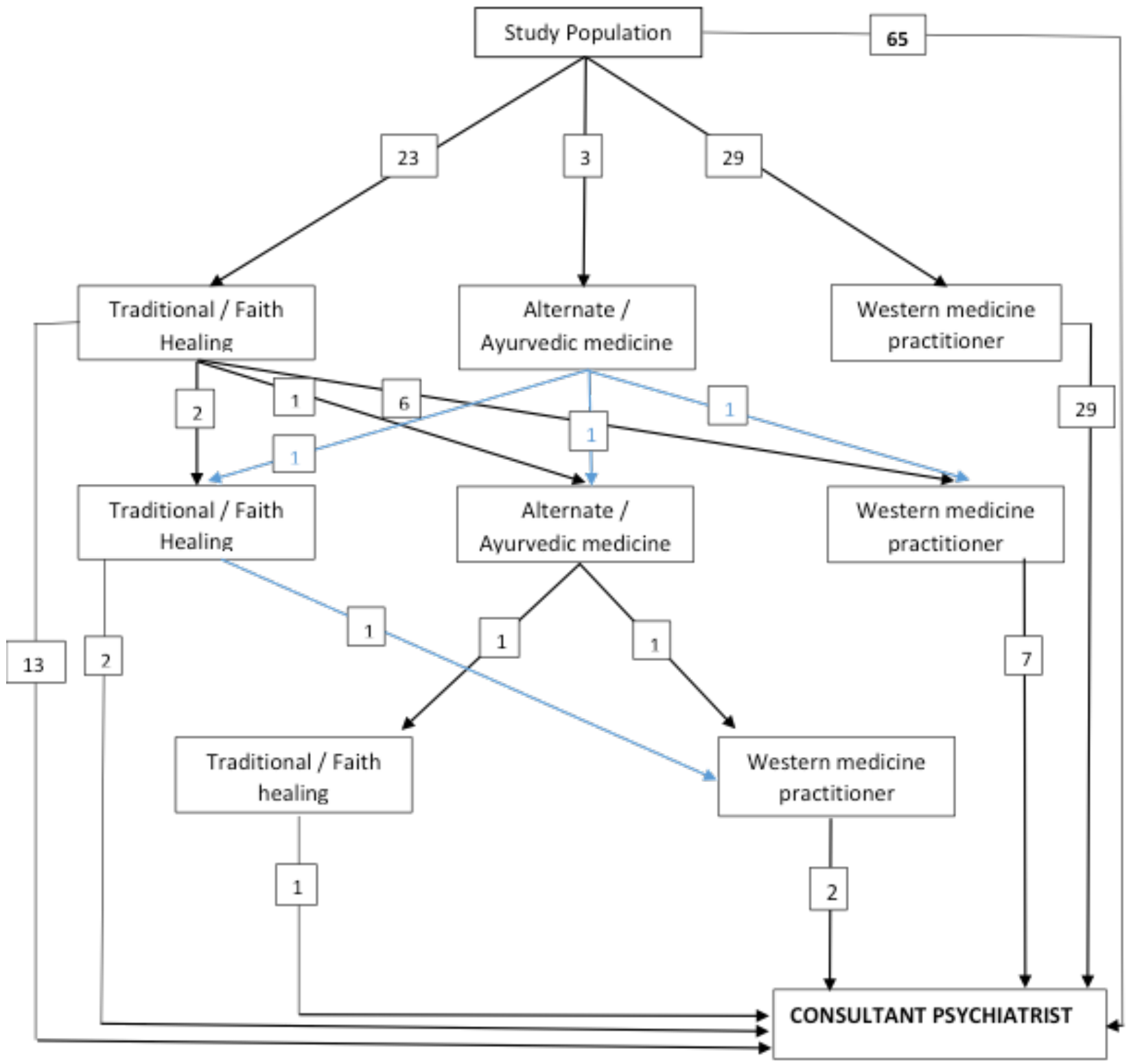

Figure 1. Flow diagram of the pathways to care of patients presenting to the psychiatry clinic 
Table 3. Reasons for delay in accessing mental health services

\begin{tabular}{ll}
\hline Reasons & Number $\mathbf{( \% )}$ \\
\hline Believes in alternative treatment & $10(12.2)$ \\
$\begin{array}{l}\text { Thinks disease was due to supernat- } \\
\text { ural causes }\end{array}$ & $7(8.5)$ \\
$\begin{array}{l}\text { Did not think symptoms were due } \\
\text { to a mental illness }\end{array}$ & $27(32.3)$ \\
$\begin{array}{l}\text { Thought symptoms were due to a } \\
\text { physical illness }\end{array}$ & $9(11.0)$ \\
$\begin{array}{l}\text { Thought symptoms would resolve } \\
\text { naturally }\end{array}$ & $17(20.8)$ \\
$\begin{array}{l}\text { Due to cost of medicine } \\
\text { Other reasons }\end{array}$ & $1(1.2)$ \\
Total & $11(13.4)$ \\
\hline
\end{tabular}

about $10 \%$ sought traditional faith healing and alternate medicine [6]. This study too was conducted in a tertiary care clinic.

The main reason for seeking psychiatric care as the first contact was due to recommendation by a family member or friend or belief that symptoms were due to a mental illness. Therefore, it is clear that family-social unit has a major role in influencing patients' help-seeking behaviour.

More than half the patients had engaged in some help-seeking behaviour within a month of first onset of symptoms. This is in contrast to what is commonly believed. This may be due to the highly selected nature of the sample. However, about a quarter of the participants had waited for more than 6 months to seek any form of help. Additionally, about $10 \%$ of the participants had taken more than 5 years to seek help from a psychiatrist. Thus there were a sizable number who either ignored their symptoms or sought alternative treatment for a considerable period.

The reasons for the delay in seeking help from a psychiatrist was because some patients sought help from traditional healers. A study done in Sri Lanka among community mental health workers reported that many people in the community were unaware about mental illness and the treatment available, and this intertwined with their traditional beliefs regarding causality led to poor help-seeking practices [4]. That study reported that patients thought that symptoms of mental illness were caused by supernatural causes such as 'Gods and devils' or possession by a spirit or due to causes such as loss of a loved one, academic failure or difficulty in gaining employment [4]. The difference in the findings may be because that study was conducted among community mental health workers who were exposed to a more diverse group of patients.

A study from Nigeria reported similar findings where $31.1 \%$ of the participants believed that their symptoms were caused by demonic or spiritual forces, while $20.8 \%$ thought that they were caused by psychological stress. A minority thought that the symptoms were due to hard drugs, poison from enemies $(8.2 \%)$ and familial problems $(2.8 \%)$. Some $(24.8 \%)$ were ignorant of the cause of illness. The high numbers of spiritualists, low socioeconomic status of the participants and low educational level are possible reasons for these findings [7].

Most alternative treatment did not result in significant financial burden However some did spend large amount of money on such treatment.

The strong belief that is prevalent in the community about the effectiveness of such therapies to treat mental illness is the main reason that patients seek such help. Because of the strong family ties which exist in the Sri Lankan culture family members often bear the cost of such treatment.

The functioning level of a majority of patients had been impaired after the onset of the illness. This was so in those who sought alternative methods of treatment as well as those who sought Western medical treatment. However, it is possible that those who are followed up in the clinic are those with chronic mental illness and therefore have impaired functioning.

This study had several limitations. The study was conducted in a tertiary care psychiatry clinic in Colombo. Thus the sample may be biased. Those who seek treatment from Western medical services are more likely to be referred to the clinic. It is possible that in the community there are a large number who seek treatment from alternative practitioners and do not seek help from mental health services. Thus the findings of this study cannot be generalized to the whole country. We also did not record the diagnosis of the patients. The help seeking behaviour of patients with minor psychiatric illnesses such as anxiety disorders may be different to those with major psychiatric illness such as schizophrenia and bipolar disorder.

\section{Acknowledgment}

This research was carried out as a component of the Community Stream undergraduate academic programme of the Faculty of Medicine, University of Colombo, Sri Lanka. We would like to thank the staff of the Community Stream for their support.

\section{Conflicts of interest}

Authors declare that there are no conflicts of interest.

\section{References}

1. World Health Organization 2015. Available at http://www.who.int/topics/mental disorders/en/ (accessed July 2015)

2. Patel V., Gender in Mental Health Research, WHO library Cataloguing-In publication data 2005. Available at http://www.who.int/gender/ documents/MentalHealthlast2.pdf (accessed July 2015) 
3. Sri Lanka, Mental Health Atlas, Department of Mental Health and Substance Abuse, World Health Organization 2011. Available at http://www.who. int/mental_health/evidence/atlas/profiles/lka_mh_ profile.pdf (accessed July 2015)

4. Samarasekare N., Lloyd M., Davies M., et al. The Stigma of Mental Illness in Sri Lanka : The Perspectives of Community Mental Health Workers 2011. Available at http://repository.rjt. ac.lk/jspui/bitstream/7013/500/1/stigma2011013. pdf (accessed July 2015)

5. Karandawala I., Traditional Treatment Methods used by psychiatric patients seeking treatment from the University Psychiatric Unit, National Hospital of Sri Lanka 2004

6. Mishra N., Nagpal S., Chadda R.K., Sood M. Help-seeking behaviour of patients with mental health problems visiting a tertiary care center in north India, Indian J Psychiatry, 2011; 53:234-238.

7. Nonye A.P., Oseloka E.C., Health seeking behaviour of mentally ill patients in Enugu, Nigeria, S Afr J Psychiatr, 2009;15: 19-22. 